\title{
SDHB immunohistochemistry: a useful tool in the diagnosis of Carney-Stratakis and Carney triad gastrointestinal stromal tumors
}

José Gaal' ${ }^{1,5}$, Constantine A Stratakis ${ }^{2,5}$, J Aidan Carney ${ }^{3}$, Evan R Ball ${ }^{2}$, Esther Korpershoek ${ }^{1}$, Maya B Lodish², Isaac Levy ${ }^{2}$, Paraskevi Xekouki², Francien H van Nederveen ${ }^{1}$, Michael A den Bakker ${ }^{1}$, Maureen O’Sullivan ${ }^{4}$, Winand NM Dinjens ${ }^{1}$ and Ronald R de Krijger ${ }^{1}$

${ }^{1}$ Department of Pathology, Josephine Nefkens Institute, Erasmus MC, University Medical Center Rotterdam, Rotterdam, The Netherlands; ${ }^{2}$ Section on Endocrinology and Genetics, Pediatric Endocrinology Training Program, Development Endocrinology Branch, National Institute of Health, Bethesda, MD, USA; ${ }^{3}$ Department of Laboratory Medicine and Pathology, Mayo Clinic, Rochester, MN, USA and ${ }^{4}$ School of Medicine, Trinity College Dublin, Department of Pathology and National Children's Research Centre, Our Lady's Children's Hospital, Crumlin, Dublin, Ireland

Mutations in the tumor suppressor genes SDHB, SDHC, and SDHD (or collectively SDHx) cause the inherited paraganglioma syndromes, characterized by pheochromocytomas and paragangliomas. However, other tumors have been associated with SDHx mutations, such as gastrointestinal stromal tumors (GISTs) specifically in the context of Carney-Stratakis syndrome. Previously, we have shown that SDHB immunohistochemistry is a reliable technique for the identification of pheochromocytomas and paragangliomas caused by SDHX mutations. We hypothesized that GISTs in patients with SDHx mutations would be negative immunohistochemically for SDHB as well. Four GISTs from patients with Carney-Stratakis syndrome and six from patients with Carney triad were investigated by SDHB immunohistochemistry. Five GISTs with KIT or PDGFRA gene mutations were used as controls. In addition, SDHB immunohistochemistry was performed on 42 apparently sporadic GISTs. In cases in which the SDHB immunohistochemistry was negative, mutational analysis of SDHB, $S D H C$, and SDHD was performed. All GISTs from patients with Carney-Stratakis syndrome and Carney triad were negative for SDHB immunohistochemically. In one patient with Carney-Stratakis syndrome, a germline SDHB mutation was found (p.Ser92Thr). The five GISTs with a KIT or PDGFRA gene mutation were all immunohistochemically positive for SDHB. Of the 42 sporadic tumors, one GIST was SDHB-negative. Mutational analysis of this tumor did not reveal an SDHx mutation. All SDHB-negative GISTs were located in the stomach, had an epithelioid morphology, and had no KIT or PDGFRA mutations. We show that Carney-Stratakis syndromeand Carney-triad-associated GISTs are negative by immunohistochemistry for SDHB in contrast to KIT- or PDGFRA-mutated GISTs and a majority of sporadic GISTs. We suggest that GISTs of epithelioid cell morphology are tested for SDHB immunohistochemically. In case of negative SDHB staining in GISTs, Carney-Stratakis syndrome or Carney triad should be considered and appropriate clinical surveillance should be instituted. Modern Pathology (2011) 24, 147-151; doi:10.1038/modpathol.2010.185; published online 1 October 2010

Keywords: Carney-Stratakis syndrome; Carney triad; gastrointestinal stromal tumor; immunohistochemistry; SDHB

Correspondence: Dr J Gaal, MD, Department of Pathology, Josephine Nefkens Institute, Erasmus MC, University Medical Center Rotterdam, PO Box 2040, Rotterdam 3000 CA, The Netherlands. E-mail: j.gaal@erasmusmc.nl

${ }^{5}$ These authors contributed equally to this work.

Received 20 May 2010; revised 3 August 2010; accepted 4 August 2010; published online 1 October 2010
Succinate dehydrogenase (SDH) is an enzyme complex that catalyzes the oxidation of succinate to fumarate in the citric acid cycle and participates in the electron transport chain. SDH is located in the mitochondrial inner membrane and consists of four nuclear-encoded subunits: the flavoprotein SDHA, the iron-sulfur protein SDHB, and the integral membrane proteins SDHC, and SDHD. ${ }^{1}$ 
Mutations in the different subunits result in very different disorders. Mutations in SDHA cause Leigh's syndrome, a rare inherited neurometabolic disorder characterized by degeneration of the central nervous system. ${ }^{2}$ Mutations in the tumor suppressor genes $S D H B, S D H C, S D H D$ from here on collectively referred to as $S D H x$, occur in patients with the pheochromocytoma-paraganglioma syndromes. ${ }^{3}$ Diverse additional tumors have been associated with SDHx mutations, including gastrointestinal stromal tumors (GISTs), renal cell carcinomas, renal oncocytomas, and, rarely, papillary thyroid carcinomas, neuroblastomas, and seminomas. ${ }^{4-8}$

Of these tumors, GISTs occur most frequently in patients with $S D H x$ mutations. The majority of sporadic GIST is caused by mutations in the KIT and PDGFRA genes whereas four different $S D H B$ mutations, two $S D H C$ mutations, and one $S D H D$ mutation have been identified in patients with the familial dyad of paraganglioma and GIST, also known as the Carney-Stratakis syndrome. ${ }^{7}$ The Carney triad is similar to Carney-Stratakis syndrome, but includes pulmonary chondromas and is apparently infrequently inherited; $S D H x$ mutations have not been described in the Carney triad. ${ }^{9}$

In a previous report, we showed that SDHB immunohistochemistry is a reliable technique to identify pheochromocytomas and paragangliomas caused by mutations in $S D H B, S D H C$, and $S D H D .{ }^{10}$ It seemed likely that other tumors in patients with SDHx mutations would be negative for SDHB immunohistochemistry, as well. In this study, we performed SDHB immunohistochemistry on GISTs that occurred as a component of the CarneyStratakis syndrome and the Carney triad. In addition, we performed SDHB immunohistochemistry on a series of apparently sporadic GISTs.

\section{Materials and methods}

\section{Tumor Samples}

Four GISTs from patients with the Carney-Stratakis syndrome and six GISTs from patients with Carney triad were available for this study. Distinguishing Carney triad and Carney-Stratakis syndrome is difficult in individual patients. In this study, we used the familial predisposition and paraganglioma as the first presenting tumor in Carney-Stratakis syndrome and the presence of pulmonary chondroma, female predominance, and GIST as the first presenting tumor in Carney triad as differentiating features, as described by Carney. ${ }^{11}$ As a control group, we used five GISTs with a mutation in KIT or PDGFRA. GIST diagnosis was made based on histology and verified immunohistochemically using DOG1 (RM-9132-R7) antibody (Thermo Scientific, Cheshire, UK; 1:50) and CD117, c-kit (A4502) antibody (DAKO, Heverlee, Belgium; 1:25).

In addition, we investigated a series of 42 formalin-fixed paraffin-embedded sporadic GISTs that were diagnosed in Erasmus MC between 2001 and 2009. These samples were anonymously used according to the code for adequate secondary use of tissue, code of conduct: 'Proper Secondary Use of Human Tissue' established by the Dutch Federation of Medical Scientific Societies (http:// www.federa.org).

\section{Immunohistochemistry}

All GISTs were investigated by SDHB immunohistochemistry as previously described, ${ }^{10}$ using the rabbit polyclonal antibody HPA002868 (SigmaAldrich, St Louis, MO, USA; 1:500). Immunoreactivity was scored independently by two observers (JG and RRdK) who were blinded to all clinical, pathological, and molecular data. Slides with a granular staining in the tumor cell cytoplasm were scored as positive. Slides in which the tumor cells were negative or showed diffuse cytoplasmatic staining, but with granular staining in endothelial cells (internal control), were scored as negative. Samples lacking the internal positive control were considered non-informative and were repeated.

\section{Mutational Analysis}

Following SDHB immunohistochemical evaluation, $S D H x$-gene mutational analysis was performed on the tumors with negative SDHB immunohistochemistry. A region containing at least $70 \%$ tumor cells was micro-dissected from the tumor block and DNA was isolated using the Puregene DNA isolation kit (Qiagen, Minneapolis, MN, USA) according to the manufacturer's protocol. No tumor DNA was available of one sample and germline DNA was used instead. Mutational analysis was performed by direct sequencing of the open reading frames, including the exon-intron boundaries of the $S D H B$, $S D H C$, and $S D H D$ genes. In addition, sequence analysis of exons $8,9,11,13$, and 17 of the KIT proto-oncogene and exons 12, 14, and 18 of the PDGFRA gene was performed on the SDHB immunonegative tumors.

\section{Results}

There was uniform agreement between the two observers in classifying tumors as SDHB-positive or SDHB-negative. Four GISTs from patients with the Carney-Stratakis syndrome were negative for SDHB by immunohistochemistry (Figure 1a). In contrast, all KIT- or PDGFRA-mutated GISTs were SDHB-positive (Figures $1 \mathrm{~b}$ and $\mathrm{c}$ ). In one of the Carney-Stratakis syndrome patients, an $S D H B$ germline mutation (p.Ser92Thr) was present. In the other three patients no mutations in $S D H B, S D H C$, and $S D H D$ were found. The six GISTs from patients with Carney triad were negative for SDHB by 
HE
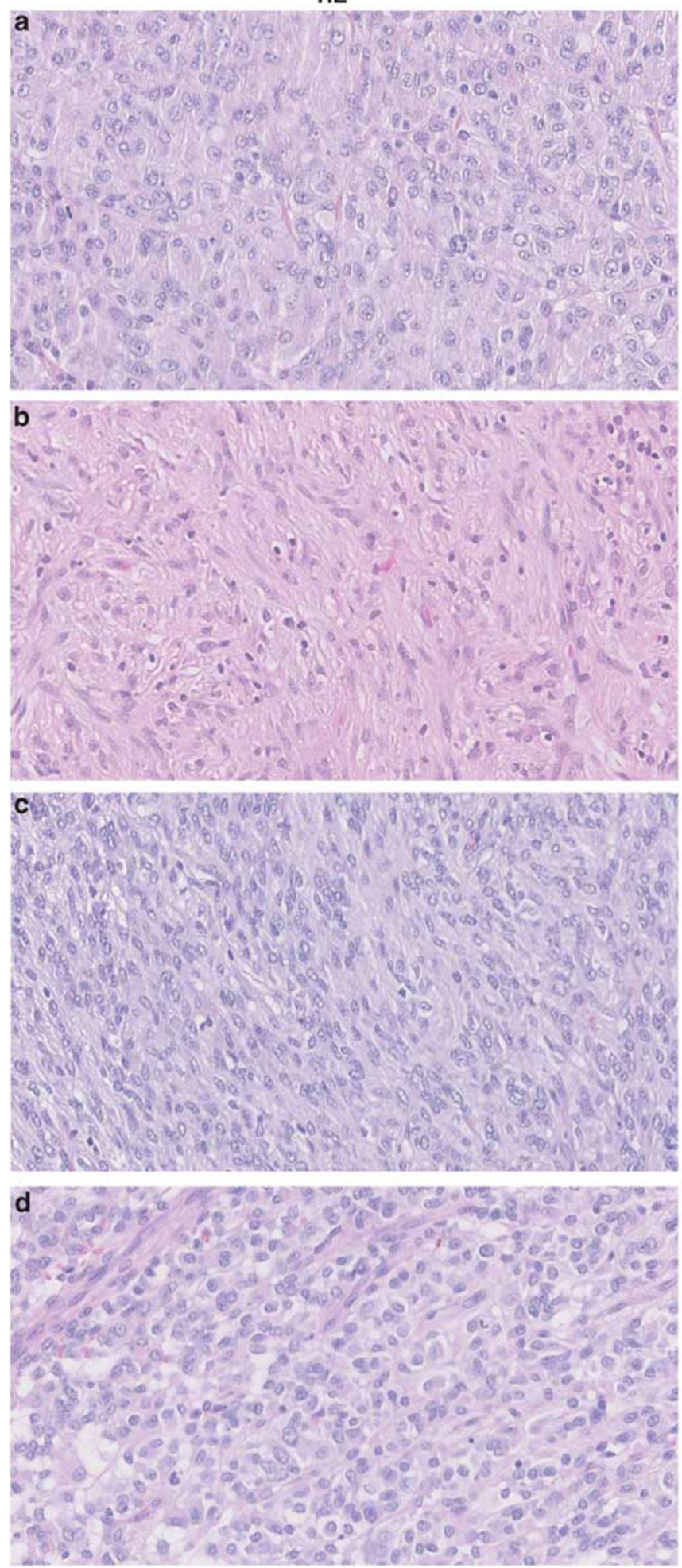

SDHB
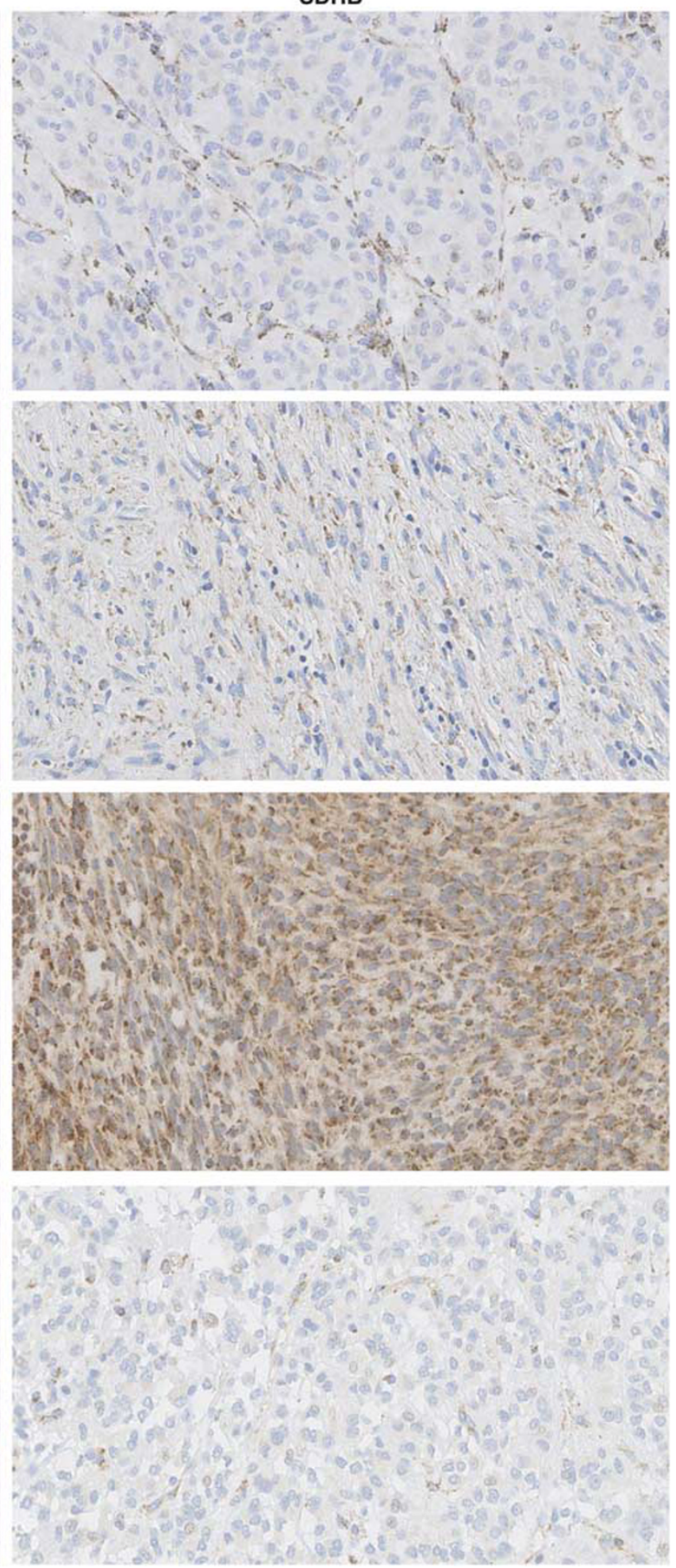

Figure 1 H\&E staining and SDHB immunohistochemistry. (a) Gastrointestinal stromal tumor from a patient with the Carney-Stratakis syndrome and negative immunostaining for SDHB. The endothelial cells serve as an internal positive control. (b) Gastrointestinal stromal tumor with a PDGFRA mutation and (c) KIT mutation. All cells show granular SDHB staining. (d) Gastrointestinal stromal tumor from a patient with Carney triad and negative immunostaining for SDHB.

immunohistochemistry (Figure 1d). However, no mutations in $S D H B, S D H C$, and $S D H D$ were found. The GISTs in the Carney-Stratakis syndrome and triad all had an epithelioid morphology. In contrast, the KIT- and PDGFRA-mutated tumors had a spindle-cell morphology. The clinical details of the 
Table 1 Details of the Carney-Stratakis syndrome and Carney triad patients

\begin{tabular}{|c|c|c|c|c|c|c|}
\hline & Sex & Age at diagnosis & Location & Cell type & Other tumors & SDHx mutation \\
\hline \multicolumn{7}{|c|}{ CSS } \\
\hline 1 & M & 18 & Stomach & Epithelioid & No & Yes \\
\hline 2 & $\mathrm{M}$ & 10 & Stomach & Epithelioid & No & No \\
\hline 3 & M & 50 & Stomach & Epithelioid & PGL & No \\
\hline 4 & $\mathrm{~F}$ & $\mathrm{U}$ & Stomach & Epithelioid & PGL, angiolipoma & No \\
\hline \multicolumn{7}{|l|}{$C T$} \\
\hline 1 & $\mathrm{~F}$ & 12 & Stomach & Epithelioid & No & No \\
\hline 2 & $\mathrm{~F}$ & 41 & Stomach & Epithelioid & PGL & No \\
\hline 3 & $\mathrm{~F}$ & 25 & Stomach & Epithelioid & No & No \\
\hline 4 & $\mathrm{~F}$ & 13 & Stomach & Epithelioid & No & No \\
\hline 5 & $\mathrm{~F}$ & 28 & Stomach & Epithelioid & Adrenal adenoma & No \\
\hline 6 & M & 51 & Stomach & Epithelioid & Chondroma & No \\
\hline
\end{tabular}

CSS: Carney-Stratakis syndrome; CT: Carney triad; SDHx: SDHB, SDHC, and SDHD gene; U: unknown, PGL: paraganglioma, GIST: gastrointestinal stromal tumor.

Carney-Stratakis syndrome and Carney-triad patients are summarized in Table 1.

Among the 42 sporadic GISTs, one GIST (2\%) from a 41-year-old woman, located in the stomach, was negative for SDHB immunohistochemically. She developed a medullary thyroid carcinoma at age 45 years with local lymph node and liver metastasis. The medullary thyroid carcinoma was positive for SDHB immunohistochemistry. Microscopy of the GIST showed an epithelioid morphology and the tumor cells were positive for CD117 and DOG1. Mutational analysis revealed no mutations in $S D H B, S D H C, S D H D$, and $S D H A F 2$ genes. Sequencing analysis of exons 8, 9, 11, 13, and 17 of the KIT proto-oncogene and exons 12, 14, and 18 of the PDGFRA gene did not reveal mutations.

\section{Discussion}

In pheochromocytomas and paragangliomas, absence of SDHB staining is an indicator of Complex II disruption caused by $S D H B, S D H C$, or $S D H D$ mutations. ${ }^{10}$ In this study, a GIST from a patient with Carney-Stratakis syndrome had a proven germline $S D H B$ mutation, which confirms our hypothesis that GISTs caused by mutations in $S D H B, S D H C$, and $S D H D$ would be negative with SDHB immunohistochemistry.

$S D H B$ mutations are also described in different types of renal tumors, ${ }^{6,8,12}$ usually clear cell carcinomas, but the mutations have also been found in oncocytomas, eosinophilic chromophobe renal cell carcinomas, and papillary renal cell carcinomas. Previously, we reported negative SDHB immunohistochemistry in a renal cell carcinoma. ${ }^{10}$ This suggests that all tumor types in patients with SDHx mutations are characterized by absent SDHB staining.

The GISTs from Carney-Stratakis syndrome and Carney triad patients in our study were negative for
SDHB by immunohistochemistry, results that are concordant with a recent publication of Gill et al, ${ }^{13}$ in which tumors are described from five Carneytriad patients with negative SDHB immunohistochemistry. However, they did not study any CarneyStratakis syndrome patients. We found that mutations in $S D H B, S D H C$, and $S D H D$ were absent in all but one SDHB-immunonegative GIST, which came from a Carney-Stratakis syndrome patient. In addition, we found no proof of loss of heterozygosity of one of the SDH genes in these tumor samples (results not shown). In a previous study of pheochromocytomas and paragangliomas, we found six tumors with negative SDHB immunohistochemistry, but lacking $S D H B, S D H C$, and $S D H D$ mutations $(11 \%)^{10,14}$ This may be due to a $<100 \%$ sensitivity of the technique of sequence analysis or to the fact that we did not perform systematic multiplex ligation-dependent probe amplification in all our samples, thus probably missing up to $10 \%$ of genetic abnormalities. However, it is also possible that epigenetic changes or other genes affect complex II, and that mutations in such additional genes might result in disruption of complex II and subsequently in negative SDHB immunohistochemistry. The question, therefore, remains whether absent SDHB immunostaining implies the presence of $S D H x$ mutations in GISTs, as we have shown with $>85 \%$ sensitivity in pheochromocytomas and paragangliomas. Although the mechanism of tumorigenesis of the SDHB immunohistochemically-negative GISTs is unknown, several studies have shown that VEGF and HIF $1 \alpha$ are relatively overexpressed in GISTs, ${ }^{15,16}$ as is the case in SDHx-mutated paragangliomas. The negative SDHB immunohistochemistry in Carney-Stratakis syndrome and Carney-triad GISTs indicates the absence of SDHB protein and functional complex II. ${ }^{10}$ The mammalian mitochondria contain about 1100 proteins of which nearly 300 are uncharacterized, so it can be anticipated that pathogenic mutations or functional abnormalities of 
other mitochondrial proteins both drive tumorigenesis and account for SDHB negativity in CarneyStratakis syndrome and Carney-triad-related GISTs.

Among the 42 apparently sporadic GISTs we studied, one tumor (2\%) was negative for SDHB by immunohistochemistry. This finding is in agreement with Gill et $a l^{13}$ who found that $3 \%$ (3/101) of sporadic GISTs in their series was negative for SDHB immunohistochemically. Interestingly, our patient developed a medullary thyroid carcinoma 4 years after diagnosis of the GIST. This combination of GIST and medullary thyroid carcinoma has been described previously in a patient with multiple endocrine neoplasia type $2 \mathrm{~A} \cdot{ }^{17}$ In our case, we found a somatic mutation in RET in the medullary thyroid carcinoma (results not shown). In addition, SDHB immunohistochemistry was positive in this medullary thyroid carcinoma, indicating that the tumor was not caused by complex II disruption.

Gill et al ${ }^{13}$ divided GISTs into two broad groups: GIST type 1, which includes most GISTs and occurs mainly in adults and KIT- or PDGFRA-mutant tumors. Type I tumors usually show spindled morphology. GIST type 2 occurs predominantly in children and young adults. Type 2 tumors show an epithelioid morphology, occur exclusively in the stomach, and are never associated with KIT or PDGFRA mutations. The one apparently sporadic SDHB-negative GIST in our study, although occurring in an adult, was located in the stomach and had an epithelioid morphology, in line with the observations by Gill et al.

In conclusion, we have shown that CarneyStratakis syndrome and Carney triad GISTs are negative for SDHB by immunohistochemistry in contrast to KIT- or PDGFRA-mutated GISTs and the majority of apparently sporadic GISTs, which are immunopositive. Our findings suggest that absent SDHB immunostaining in GISTs has a high likelihood of a syndromic diagnosis of either CarneyStratakis syndrome or the Carney triad. Consequently, $S D H x$ mutational analysis and clinical surveillance for the development of paragangliomas and pulmonary chondromas should be instituted.

\section{Acknowledgements}

We thank Frank van der Panne for assistance with preparation of the figure. This work was supported in part by the Intramural Program of the Eunice Kennedy Shriver National Institute of Child Health \& Human Development. The work described in this article was supported by a grant from Erasmus MC.

\section{Disclosure/conflict of interest}

The authors declare no conflict of interest.

\section{References}

1 Lancaster CR. Succinate:quinone oxidoreductases: an overview. Biochim Biophys Acta 2002;1553:1-6.

2 Parfait B, Chretien D, Rotig A, et al. Compound heterozygous mutations in the flavoprotein gene of the respiratory chain complex II in a patient with Leigh syndrome. Hum Genet 2000;106:236-243.

3 Lenders JW, Eisenhofer G, Mannelli M, Pacak K. Phaeochromocytoma. Lancet 2005;366:665-675.

4 Cascon A, Landa I, Lopez-Jimenez E, et al. Molecular characterisation of a common SDHB deletion in paraganglioma patients. J Med Genet 2008;45:233-238.

5 Galera-Ruiz H, Gonzalez-Campora R, Rey-Barrera M, et al. W43X SDHD mutation in sporadic head and neck paraganglioma. Anal Quant Cytol Histol 2008;30:119-123.

6 Neumann HP, Pawlu C, Peczkowska M, et al. Distinct clinical features of paraganglioma syndromes associated with SDHB and SDHD gene mutations. JAMA 2004;292:943-951.

7 Pasini B, McWhinney SR, Bei T, et al. Clinical and molecular genetics of patients with the Carney-Stratakis syndrome and germline mutations of the genes coding for the succinate dehydrogenase subunits SDHB, SDHC, and SDHD. Eur J Hum Genet 2008;16:79-88.

8 Srirangalingam U, Walker L, Khoo B, et al. Clinical manifestations of familial paraganglioma and phaeochromocytomas in succinate dehydrogenase B (SDH-B) gene mutation carriers. Clin Endocrinol (Oxf) 2008;69:587-596.

9 Matyakhina L, Bei TA, McWhinney SR, et al. Genetics of Carney triad: recurrent losses at chromosome 1 but lack of germline mutations in genes associated with paragangliomas and gastrointestinal stromal tumors. J Clin Endocrinol Metab 2007;92:2938-2943.

10 van Nederveen FH, Gaal J, Favier J, et al. An immunohistochemical procedure to detect patients with paraganglioma and phaeochromocytoma with germline SDHB, SDHC, or SDHD gene mutations: a retrospective and prospective analysis. Lancet Oncol 2009;10:764-771.

11 Carney JA. Carney triad: a syndrome featuring paraganglionic, adrenocortical, and possibly other endocrine tumors. J Clin Endocrinol Metab 2009;94:3656-3662.

12 Ricketts C, Woodward ER, Killick P, et al. Germline SDHB mutations and familial renal cell carcinoma. J Natl Cancer Inst 2008;100:1260-1262.

13 Gill AJ, Chou A, Vilain R, et al. Immunohistochemistry for SDHB divides gastrointestinal stromal tumors (GISTs) into 2 distinct types. Am J Surg Pathol 2010;41:805-814.

14 Gill AJ, Benn DE, Chou A, et al. Immunohistochemistry for SDHB triages genetic testing of SDHB, SDHC, and SDHD in paraganglioma-pheochromocytoma syndromes. Hum Pathol 2010;34:636-644.

15 Takahashi R, Tanaka S, Hiyama T, et al. Hypoxiainducible factor-1alpha expression and angiogenesis in gastrointestinal stromal tumor of the stomach. Oncol Rep 2003;10:797-802.

16 Takahashi R, Tanaka S, Kitadai Y, et al. Expression of vascular endothelial growth factor and angiogenesis in gastrointestinal stromal tumor of the stomach. Oncology 64:266-274.

17 A gastrointestinal stromal tumor in a patient with multiple endocrine neoplasia type $2 \mathrm{~A}$ and metastatic medullary thyroid cancer to the ovaries. Endocr Pract 2008;14:898-901. 